\title{
Islet-1 induces the differentiation of mesenchymal stem cells into cardiomyocyte-like cells through the regulation of Gen5 and DNMT-1
}

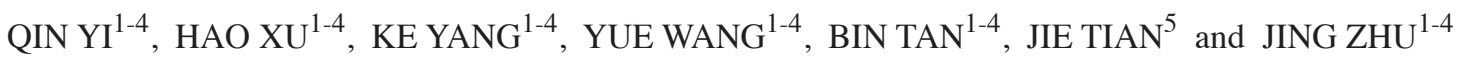 \\ ${ }^{1}$ Department of Pediatric Research Institute, Children's Hospital of Chongqing Medical University; ${ }^{2}$ Ministry of Education \\ Key Laboratory of Child Development and Disorders; ${ }^{3}$ China International Science and Technology Cooperation Base \\ of Child Development and Critical Disorders; ${ }^{4}$ Chongqing Key Laboratory of Pediatrics; ${ }^{5}$ Cardiovascular Department \\ (Internal Medicine), Children's Hospital of Chongqing Medical University, Chongqing 400014, P.R. China
}

Received January 5, 2016; Accepted February 9, 2017

DOI: $10.3892 / \mathrm{mmr} .2017 .6343$

\begin{abstract}
Previous studies from this group demonstrated that insulin gene enhancer binding protein ISL-1 (Islet-1) specifically induces the differentiation of mesenchymal stem cells (MSCs) into cardiomyocyte-like cells through histone acetylation. However, the underlying mechanisms remain unclear. In the present study, the role of the histone acetylation and DNA methylation on the regulatory mechanism of the Islet-1 was further investigated by methylation-specific polymerase chain reaction (PCR), chromatin immunoprecipitation quantitative PCR and western blot analysis. The results demonstrated that Islet-1 upregulated expression of general control of amino acid biosynthesis protein 5 (Gcn5) and enhanced the binding of Gen 5 to the promoters of GATA binding protein 4 (GATA4) and NK2 homeobox 5 (Nkx2.5). In addition, Islet-1 downregulated DNA methyltransferase (DNMT)-1 expression and reduced its binding to the GATA4 promoter. In contrast, the amount of DNMT-1 binding on Nkx2.5 did not match the expression trend. Therefore, it was concluded that Islet-1
\end{abstract}

Correspondence to: Professor Jing Zhu, Department of Pediatric Research Institute, Children's Hospital of Chongqing Medical University, 2 Zhongshan Road, Yuzhong, Chongqing 400014, P.R. China

E-mail: 1686598427@qq.com

Abbreviations: MSCs, mesenchymal stem cells; LIM-HD, LIM-homeodomain; DNMT, DNA methyltransferase; HATs, histone acetyltransferases; cTnT, troponin T2 cardiac type; ChIP, chromatin immunoprecipitation; MSP, methylation-specific PCR; Gen5, general control of amino acid biosynthesis protein 5; Islet-1, insulin gene enhancer binding protein ISL-1; H3K9, histone H3 at lysine 9; GATA4, GATA binding protein 4; Nkx2.5, NK2 homeobox 5; Mef2c, myocyte enhancer factor 2C; Lv, lentivirus; SDS-PAGE, sodium dodecyl sulfate polyacrylamide gel electrophoresis

Key words: Islet-1, DNA methylation, histone acetylation, mesenchymal stem cell, cardiomyocyte may influence the histone acetylation and DNA methylation of GATA4 promoter region via Gen5 and DNMT-1 during the MSC differentiation into cardiomyocyte-like cells, thus prompting the expression of GATA4. The Nkx2.5 was likely only affected by histone acetylation instead of DNA methylation. The present study demonstrated that Islet-1 induces the differentiation of mesenchymal stem cells into cardiomyocyte-like cells through a specific interaction between histone acetylation and DNA methylation on regulating GATA4.

\section{Introduction}

The human heart loses most of its regenerative capacity during postnatal development, and is not able to replace any defects after damage with functional myocardium (1). An increasing number of general treatments have been reported to be unable to aid complete cardiac regeneration and repair (2). Since stem cells can be induced for specific differentiation into cardiomyocytes under certain conditions, therapies based on stem cells have generated interest among researchers in recent years $(3,4)$. Mesenchymal stem cells (MSCs) have the characteristics of autologous transplantation, as they are easy to isolate and are characterized by a strong ability for amplification, excellent gene stability and low immunogenicity $(1,5)$. A large number of studies have shown that cardiovascular regeneration based on stem cells may cure cardiovascular diseases with cardiomyocyte damage (6). However, the specific molecular mechanism underlying this cure remains elusive.

Insulin gene enhancer binding protein ISL-1 (Islet-1), a subtype of the LIM-homeodomain (LIM-HD) transcription factor subfamily, contains one DNA binding site and two LIM domains (7-9). Several studies have demonstrated that Islet-1 is crucial to cardiac development and cardiomyocyte differentiation $(10,11)$. Islet1-null mice completely lack the outflow tract, right ventricle and much of the atria $(10,12)$. Lineage tracing of Islet1-expressing progenitors demonstrate that Islet-1 is a marker for a distinct population of undifferentiated cardiac progenitors (12). Previous studies from this group indicated that Islet-1 serves a critical role in the differentiation of MSCs 
into cardiomyocytes and promotes the expression of heart development-related genes in MSCs.

Islet-1 may be able to affect the acetylation levels of the cardiomyocyte-specific early transcription factors NK2 homeobox 5 (Nkx2.5) and GATA binding protein 4 (GATA4) to regulate their expression levels and promote their differentiation into cardiomyocytes (13). Although previous studies have demonstrated that histone acetyltransferases (HATs) serve critical roles in the regulation of cardiomyocyte-specific gene expression $(14,15)$, the specific HATs involved in this process are unknown. In addition to histone acetylation, DNA methylation is significant in the regulation of gene expression (16). For example, DNA methyltransferase (DNMT)-1, DNMT-3a and DNMT-3b participate as the major DNMTs in the methylation of different genes to regulate their DNMTs in the methylation of different genes to regulate their expression (17-20). Therefore, it was hypothesized that DNA methylation participated in the regulation of relevant genes during the complex process of MSCs differentiation into cardiomyocytes. However, few investigations have been conducted to identify the major DNMTs involved in this process.

In the current study, C3H10T1/2 MSCs were infected with lentiviruses overexpressing Islet-1, in order to promote their specific differentiation into cardiomyocytes. Alterations over time in histone $\mathrm{H} 3 \mathrm{~K} 9$ acetylation and DNA methylation levels on the promoter regions of GATA4 and Nkx2.5 were assessed during the process of MSC differentiation into cardiomyocytes. In addition, HATs and DNMTs that bound to the GATA4 and Nkx 2.5 promoter regions were evaluated. The expression trends of the early-stage cardiomyocyte-specific genes GATA4 and Nkx2.5 were examined and the relationship between the changing trends in histone H3K9 acetylation levels and DNA methylation levels during the MSCs differentiation into cardiomyocyte-like cells promoted by Islet-1. Finally, a mechanism underlying the involvement of these two epigenetic modifications in the regulation of differentiation was preliminarily proposed.

\section{Materials and methods}

Cell culture and lentiviral vector infection. C3H10T1/2 cells, obtained from University of Chicago Molecular Oncology Laboratory (Chicago, IL, USA), were grown in Dulbecco's modified Eagle's medium (Gibco; Thermo Fisher Scientific, Inc., Waltham, MA, USA) supplemented with $10 \%$ fetal bovine serum (FBS, EMD Millipore, Billerica, MA, USA). Lentiviruses (Lv) overexpressing GFP or Islet-1/GFP (multiplicity of infection=20) (GeneChem Co., Ltd., Shanghai, China), and $5 \mu \mathrm{g} / \mathrm{ml}$ polybrene (GeneChem Co., Ltd.) were mixed together and added to the culture medium of cells when they reached $30 \%$ confluence. The culture medium was replaced following culturing at $37^{\circ} \mathrm{C}$ in $5 \% \mathrm{CO}_{2}$ for $24 \mathrm{~h}$. Fluorescence microscopy (Eclipse Ti-s; Nikon Corporation, Tokyo, Japan) was used to observe the GFP expression after 3 days. The infection efficiency was assessed with flow cytometry (BD FACSCanto II, BD Biosciences, San Jose, CA, USA), and prepared by BD FACS Diva version 3.0. The experiment was divided into 3 groups: The blank group, the Lv-GFP group (GFP cells) and the Lv-islet-1 group (cells infected with a plasmid overexpressing Islet-1/GFP). The Lv-islet-1 group was further divided into the following subgroups: Islet-1-1 week, Islet-1-2 weeks, Islet-1-3 weeks and Islet-1-4 weeks based on the lentiviral infection time.

Immunofluorescence. C3H10T1/2 cells ( $1 \times 10^{5}$ cells/well) were plated in 24 -well plates on $1 \times 1 \mathrm{~cm}^{2}$ glass coverslips. Then, the cells were fixed in absolute acetone for $15 \mathrm{~min}$ at $4^{\circ} \mathrm{C}$. Following 3 washes with PBS, the cells on the glass coverslips were blocked with goat serum (dilution, 1:20, ZSGB-BIO, Beijing, China), washed again, and incubated with the primary anti-cardiac troponin $\mathrm{T}(\mathrm{cTnT})$ monclonal antibody (ab209813; 1:400; Abcam, Cambridge, MA, USA) overnight at $4^{\circ} \mathrm{C}$. Then, the cells were washed with PBS and incubated with a Cy3-conjugated secondary antibody (CW0159S; 1:150; Beijing Cowin Bioscience Co., Ltd., Beijing, China) for $1 \mathrm{~h}$ at $37^{\circ} \mathrm{C}$. Following washing with PBS, 4',6-diamidino-2-phenylindole was added for $3 \mathrm{~min}$. Following the final wash, images were acquired under a fluorescence microscope (BX51; Olympus Corporation, Tokyo, Japan) and prepared by Nikon NIS-element AR 4.0 software. A total of six fields of view were assessed, and three replicates were performed.

Total RNA extraction and reverse transcription-quantitative polymerase chain reaction $(R T-q P C R)$. Cellular RNA was extracted from the blank group, the Lv-GFP group and the Lv-islet-1 groups (Islet-1-1, Islet-1-2, Islet-1-3 and Islet-1-4 weeks) according to the instructions of the RNA extraction reagent kit (RP120; BioTeke Corporation, Beijing, China) and subjected to reverse transcription by PrimeScript ${ }^{\mathrm{TM}}$ RT Master Mix kit (RR047A; Takara Biotechnology Co., Ltd., Dalian, China). The cDNA was amplified (RR047A; Takara Biotechnology Co., Ltd.), using the reaction conditions of: 40 cycles of $95^{\circ} \mathrm{C}$ for $30 \mathrm{sec}, 95^{\circ} \mathrm{C}$ for $5 \mathrm{sec}$ and $60^{\circ} \mathrm{C}$ for $40 \mathrm{sec}$. Each reaction contained one blank well, and the samples of each group included three replicate wells. $\beta$-actin was used as the internal control. The relative expression levels of the genes were calculated using the $2^{-\Delta \Delta \mathrm{Cq}}$ method (21). The changes in the gene expression levels of GATA4, Nkx2.5 and Mef2c were assessed at all time points. The primer sequences of the genes are provided in Table I.

Chromatin immunoprecipitation (ChIP)-qPCR assay. Formaldehyde (1\%) was added to the samples to cross-link the protein-DNA complexes. The ChIP trials were conducted using a ChIP assay kit (Merck KGaA Darmstadt, Germany). Following cross-linking, the DNA was fragmented by sonication (Bioruptor UCD-200; Diagenode, Liège, Belgium) consisting of 25 cycles of $30 \mathrm{sec}$ each, with an interval of $30 \mathrm{sec}$ to cool down. Then, the protein-DNA complexes were precipitated with the following antibodies: Histone H3 (acetyl $\mathrm{K} 9$; ab10812; $3 \mu \mathrm{g} / \mu \mathrm{l}$ ), general control of amino acid biosynthesis protein 5 (Gcn5; ab18381; $7 \mu \mathrm{g} / \mu \mathrm{l}), \mathrm{P} 300$ (ab14984; $5 \mu \mathrm{g} / \mu \mathrm{l})$, DNMT1 (ab87656; $5 \mu \mathrm{g} / \mu \mathrm{l})$, DNMT3a (ab2850; $9 \mu \mathrm{g} / \mu \mathrm{l})$ or DNMT3b (ab2851; $9 \mu \mathrm{g} / \mu \mathrm{l})$, All antibodies purchased from Abcam and incubated overnight on a shaker at $4^{\circ} \mathrm{C}$. DNA was extracted using the ChIP assay kit. The experiment included both a positive control (DNA precipitated by the RNA polymerase II antibody) and a negative control (DNA precipitated by normal mouse $\operatorname{IgG}$ ), these antibodies all part of the assay kit noted above and used according to 
Table I. Primer sequences used in reverse transcriptionquantitative polymerase chain reaction.

\begin{tabular}{ll}
\hline Target & \multicolumn{1}{c}{ Sequence (5'-3') } \\
\hline Nkx2.5 & F-GAGCCTGGTAGGGAAAGAGC \\
& R-GGTGGGTGTGAAATCTGAGG \\
GATA4 & F-GACTACCACCACCACGCTGT \\
& R-ATTCAGGTTCTTGGGCTTCC \\
Mef2c & F-ATCCCAGTGTCCAGCCATAA \\
& R-AGACCGCCTGTGTTACCTG \\
$\beta$-actin & F-GGAGATTACTGCCCTGGCTCCTA \\
& R-GACTCATCGTACTCCTGCTTGCTG
\end{tabular}

F, forward; R, reverse; Nkx2.5, NK2 homeobox 5; GATA4, GATA binding protein 4 ; Mef2c, myocyte enhancer factor $2 \mathrm{C}$.

Table II. Primer sequences and annealing temperatures used in chromatin immunoprecipitation-quantitative polymerase chain reaction.

\begin{tabular}{llc}
\hline Target & \multicolumn{1}{c}{ Sequence $\left(5^{\prime}-3^{\prime}\right)$} & $\mathrm{T}_{\mathrm{m}}\left({ }^{\circ} \mathrm{C}\right)$ \\
\hline \multirow{2}{*}{ Nkx2.5 } & F-ACCGCCTGGGTGATAGAC & 58.37 \\
& R-CCCTCCCGAGATTGAAGAT & 55.87 \\
GATA4 & F-GCTACAGGGAGTGATGAGAAG & 53.90 \\
& R-CACCAGCCCAGGAGTTTAT & 54.70
\end{tabular}

$\mathrm{T}_{\mathrm{m}}$, melting temperature; $\mathrm{F}$, forward; $\mathrm{R}$, reverse; Nkx2.5, NK2 homeobox 5; GATA4, GATA binding protein 4.

the manufacturer's protocols. The amount of extracted DNA was determined by qPCR (RR420A; Takara Biotechnology Co., Ltd., Dalian, China), the thermocycler conditions and the method of quantification strictly followed the ChIP assay kit protocols. The primer sequences and annealing temperatures of the ChIP-qPCR reaction are presented in Table II.

Methylation-specific (MS-)PCR. Cellular DNA was extracted from the blank group, the negative control group and the Lv-islet-1 groups (Islet-1-1, Islet-1-2, Islet-1-3, and Islet-1-4 weeks) according to the instructions of the DNA extraction kit (Tiangen Biotech Co., Ltd., Beijing, China). The DNA concentrations were determined to ensure that the amount of DNA treated with bisulfite was $350 \mathrm{ng}$. The volume of the DNA required for each group was calculated based on the concentration. The DNA bisulfite treatment reagent kit (Zymo Research, Irvine, CA, USA) was used. The primers for MS-PCR were designed using the MethPrimer software according to the previously described method (22). The primer sequences for GATA4 and Nkx2.5 are provided in Table III.

A 2\% agarose gel was prepared, and the loading volume of the DNA ladder marker and the MS-PCR products was $5 \mu 1$. The electrophoresis was run for $45 \mathrm{~min}$ at $120 \mathrm{~V}$ in $0.25 \mathrm{X}$ TAE agarose gel electrophoresis buffer. The agarose electrophoresis results were observed using the chemiluminescence gel imaging system (G:box; Syngene, Cambridge, UK). The gray value of the electrophoresis band was determined using the Quantity One software (version 4.6.2; Bio-Rad Laboratories, Inc., Hercules, CA, USA).

Protein extraction and western blotting. Proteins were extracted from the cells using the radioimmunoprecipitation assay reagent (P0013B; Beyotime Institute of Biotechnology, Shanghai, China) containing 1\% phenylmethanesulfonyl fluoride (cat. no. ST506; Beyotime Institute of Biotechnology) to prevent protein degradation. The protein concentrations were measured with the bicinchoninic acid method. The protein samples ( $40 \mu \mathrm{g}$ protein each well) were mixed with 5X SDS-PAGE buffer (Beyotime Institute of Biotechnology). The sample loading buffer was boiled for 5 min prior to loading onto a $10 \%$ SDS-PAGE gel. Following electrophoresis, the proteins were transferred to polyvinylidene fluoride membranes (EMD Millipore). The membranes were cut according to the marker and incubated in 5\% non-fat milk with PBS and Tween-20 (PBST, 0.05\% Tween-20) for $1 \mathrm{~h}$ on a shaker at room temperature to block non-specific protein binding. The primary antibodies used in the present study were as follows: Anti-Islet-1 (EP4182; 1:2,000; Epitomics, Burlingame, CA, USA), anti-Gen5 (ab18381; 1:1,000; Abcam), anti-P300 (ab14984; 1:1,000; Abcam), anti-DNMT1 (ab87656; 1:1,000; Abcam), anti-DNMT3a (ab2850; 1:1,000; Abcam) and anti-DNMT3b (ab2851; 1:1,000; Abcam). The $\beta$-actin antibody (A5441; 1:2,000; Sigma-Aldrich; Merck KGaA) was used as a control. The membranes were incubated with the primary antibodies overnight at $4^{\circ} \mathrm{C}$ and then washed in PBST 3 times for $10 \mathrm{~min}$ and incubated with the corresponding secondary antibody (ZB-2301 and ZB-2305, 1:2,000; ZSGB-BIO) on a shaker at room temperature. Positive bands were detected using a chemiluminescent reaction (EMD Millipore). The image collection and densitometry analyses were performed with the Quantity One analysis software (version 4.6.2; Bio-Rad Laboratories, Inc.).

Statistical analysis. Each experiment was repeated at least three times. All data were expressed as the mean \pm standard deviation. The statistical evaluations were performed using independent samples by using Student's paired t-tests, continuity correction chi-square test and one-way analysis of variance and Dunnett's as a post hoc test. SPSS software (version 17.0; SPSS Inc., Chicago, IL, USA) was used for the statistical analysis. $\mathrm{P}<0.05$ was considered to indicate a statistically significant difference.

\section{Results}

Islet-1 promotes the differentiation of MSCs into cardiomyocyte-like cells. The GFP fluorescence results demonstrated that the GFP was stably expressed, indicating that the lentiviral infection was successful (Fig. 1A). The flow cytometry results demonstrated that the infection efficiency reached $91.7 \%$ (Fig. 1B). These results ensured the reliability of subsequent experiments. The western blotting results indicated that the $\mathrm{C} 3 \mathrm{H} 10 \mathrm{~T} 1 / 2$ cells had a high level of Islet-1 expression following lentiviral infection compared with the blank group and the control group (Fig. 1C). No visible difference in morphology 
Table III. Primer sequences for GATA4 and Nkx2.5 used in methylation-specific-polymerase chain reaction.

Target

Primer

Upstream primer (5'-3')

Downstream primer $\left(5^{\prime}-3^{\prime}\right)$

Product

length

GATA4 Methylation

GGGTTTATAGGTATTGACGTCGA

GATAAAAACTACAAAACGCCGAA

(bp)

GATA4 Non-methylation AGgGTTTATAGGTATTGATGTTGA

Nkx2.5 Methylation ATTTTTTAAATTGTTATCGCGATTC

Nkx2.5 Non-methylation TTTTTAAATTGTTATTGTGATTTGT

CCAATAAAAACTACAAAACACCAAA

291

AАCCTAАСТTAAАACCCTCCCG

294

ACCTAАCTTAAAACCCTCCCAAA

203

200

GATA4, GATA binding protein 4; Nkx2.5, NK2 homeobox 5.

A

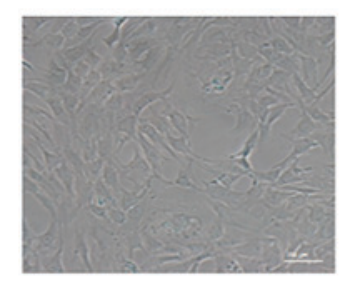

B

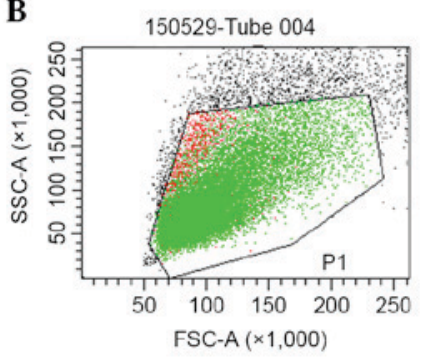

\begin{tabular}{|lccc|}
\hline Tube: tube 004 & & & \\
Population & \#Events & \%Parent & \% Total \\
All events & 30,000 & \#\#\# & 100.0 \\
PP1 & 27,502 & 91.7 & 91.7 \\
\hline GFP & 25,213 & 91.7 & 84.0 \\
\hline
\end{tabular}

C

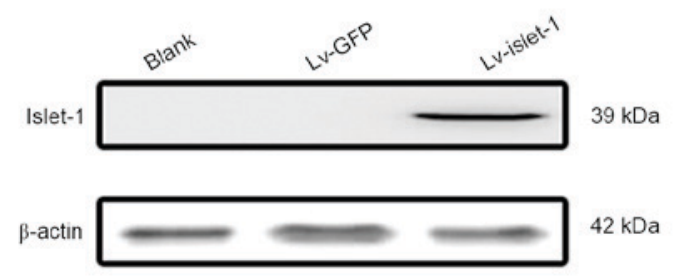

Figure 1. Successful establishment of Islet-1 overexpression model in C3H10T1/2 cells. (A) Fluorescence microscopy. Scale bar $=100 \mu \mathrm{m}$. (B) Infection efficiency, as GFP detected by flow cytometry, was $91.7 \%$. (C) Islet-1 protein expression detected by western blotting, with $\beta$-actin as a loading control. Islet-1, insulin gene enhancer binding protein ISL-1; GFP, green fluorescent protein.

was observed in untransfected MSCs and the Lv-GFP group (Fig. 2A). However, following Islet-1 transfection, the MSCs became fibroblast-like cells arranged in the same direction, exhibiting a short rod-shaped morphology and had a homogenous direction, a tight arrangement and a strong refraction (Fig. 2A). cTnT immunofluorescence was visibly higher in the Lv-islet-1 group compared with the blank and Lv-GFP groups, indicating that the MSCs expressed the cardiomyocyte-specific protein in the cytoplasm at 4 weeks following Islet-1 infection (Fig. 2B). The detection of cardiomyocyte-specific early-stage transcription factors indicated that the expression of $\mathrm{Nkx} 2.5$,
GATA4 and myocyte enhancer factor 2C (Mef2c) gradually increased with time, and was highest in the Islet-1-3W group (Fig. 2C). These results suggested that Islet-1 promoted the differentiation of MSCs into cardiomyocyte-like cells.

Histone acetylation and DNA methylation participate in the regulation of early-stage transcription factors involved in cardiomyocyte development during MSC differentiation into cardiomyocyte-like cells. The MS-PCR results indicated that the methylation level of the $\mathrm{CpG}$ sites on the GATA4 promoter gradually decreased following Islet-1 transfection; the decrease was most significant at week 3 ( $\mathrm{P}<0.05$; Fig. $3 \mathrm{~A})$. The methylation levels of the $\mathrm{CpG}$ sites on the $\mathrm{Nkx} 2.5$ gene promoter were higher but did not exhibit significant differences compared with the blank group and Lv-GFP group (Fig. 3B).

The ChIP-qPCR results demonstrated that the levels of histone acetylation on the promoter regions of GATA4 and $\mathrm{Nkx} 2.5$ in the Lv-islet-1 group were gradually increased with time; the expression of GATA4 and Nkx2.5 combined with $\mathrm{H} 3 \mathrm{~K} 9 \mathrm{ac}$ in the C3H10T1/2 cells infected with Lv-Islet-1 gradually increased, with the peak time of binding at week 3 $(\mathrm{P}<0.05$; Fig. 3C). These results indicated that histone acetylation participated in the regulation of GATA4 and Nkx2.5; by contrast, Nkx2.5 may not be affected by DNA methylation.

Islet-1 alters the histone acetylation levels of GATA4 and $N k \times 2.5$ through the regulation of Gcn5. To elucidate the mechanism underlying the involvement of histones in the regulation of early-stage transcription factors in cardiomyocytes, protein expression of the major HATs, Gcn5 and P300, was assessed. The western blotting results indicated that the expression level of Gcn5 gradually increased following Islet-1 infection: The expression levels at all time points in the Lv-islet-1 group were higher than those in the blank group and Lv-GFP group, and the Islet-1-3 W was highest (Fig. 4A). The expression levels of P300 gradually decreased and the expression levels at all time points in the Lv-islet-1 group were significantly lower than those in the blank group and the negative control group $(\mathrm{P}<0.05$; Fig. 4A).

The ChIP-qPCR results demonstrated that the levels of GATA4 and Nkx2.5 bound with Gen5 gradually increased following Islet-1 infection, which was consistent with the increased expression of Gcn5 (Fig. 4B). The binding levels at all time points in the Lv-islet-1 group were higher than those in the blank group and the Lv-GFP group $(\mathrm{P}<0.05$; Fig. 4B). The 
A
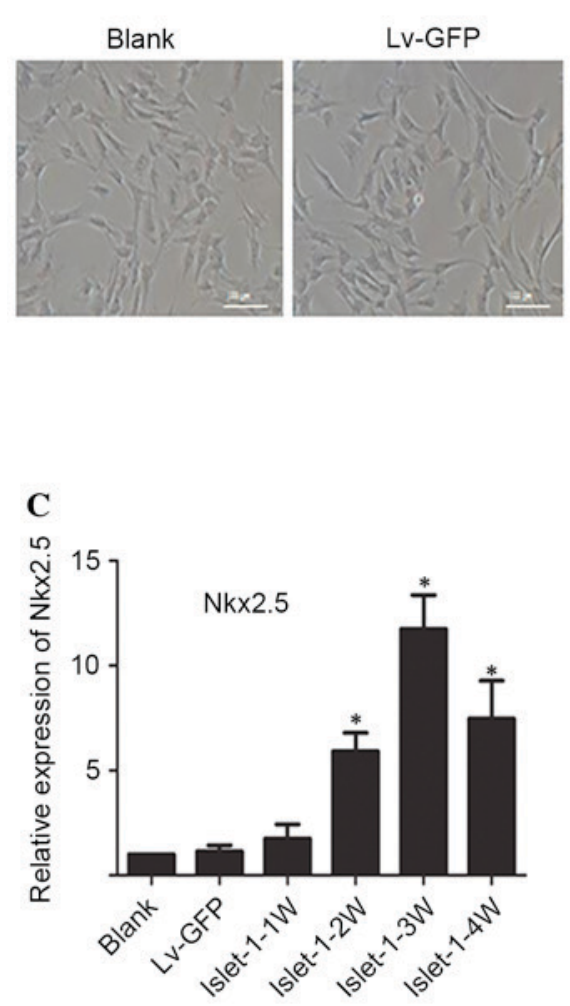

B

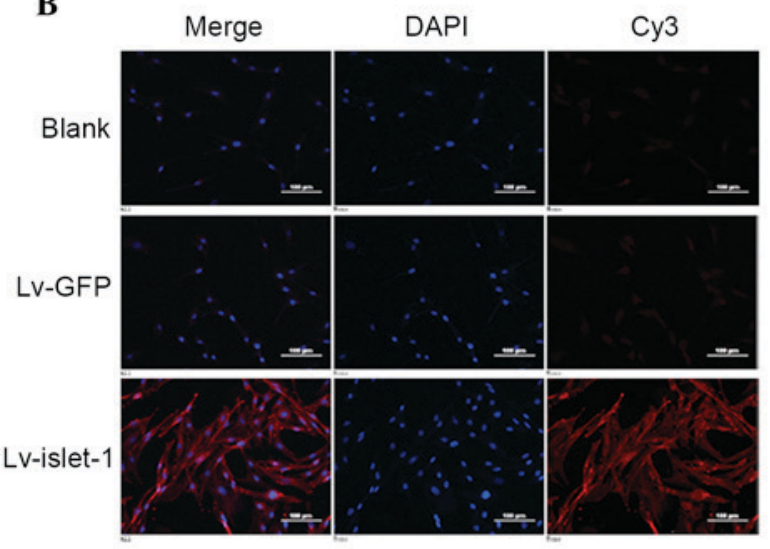

Figure 2. Islet-1 induces the differentiation of C3H10T1/2 cells into cardiomyocytes. (A) The morphological alterations in C3H10T1/2 cells transfected with Lv-GFP or Lv-islet-1 were observed under a microscope. Scale bar=100 $\mu \mathrm{m}$. (B) Expression of cTnT detected by immunofluorescence microscopy. Scale bar $=100 \mu \mathrm{m}$. (C) Reverse transcription-quantitative polymerase chain reaction detected variations in mRNA expression levels of cardiac-specific transcription factors in C3H10T1/2 cells infected with lentiviral vectors containing Islet-1. * $\mathrm{P}<0.05$ vs. blank group. Lv-GFP, lentiviral vector containing green fluorescent protein; Lv-islet-1, lentiviral vector containing Islet-1; cTnT, troponin T2 cardiac type; Nkx2.5, NK2 homeobox 5; GATA4, GATA binding protein 4; Mef2c, myocyte enhancer factor 2C; $1 \mathrm{~W}, 1$ week; $2 \mathrm{~W}, 2$ weeks; $3 \mathrm{~W}, 3$ weeks; 4 W, 4 weeks.

expression of the GATA4 and Nkx2.5 binding with P300 did not significantly change following Islet-1 infection compared with those in the blank group and the Lv-GFP group ( $\mathrm{P}>0.05$; Fig. 4C). These results indicated that Islet-1 enhanced the binding level of Gen 5 to the GATA4 and Nkx2.5 promoter regions through the increase in Gcn5 expression.

Islet-1 alters the DNA methylation levels of the GATA4 promoter region through the regulation of DNMT-1. Previous studies indicated that DNA methylation participated in the Islet-1-induced MSCs differentiation into cardiomyocyte-like cells (23). Therefore, the present study further investigated the underlying mechanism. The western blotting results indicated that the DNMT-1 expression level gradually decreased following Islet-1 infection and that the expression levels at all time points in the Lv-islet-1 group were lower than those in the blank group and the Lv-GFP group (Fig. 5A). The expression level of DNMT-3a in the Lv-islet-1 group gradually increased; the expression levels at all time points in the Lv-islet-1 group were significantly higher than those in the blank group and the Lv-GFP group ( $\mathrm{P}<0.05$; Fig. 5A). By contrast, DNMT-3b expression was almost undetectable (data not shown).

ChIP-qPCR analysis identified that the levels of GATA4 bound with DNMT-1 gradually decreased following Islet-1 infection and the binding levels at all time points in the experimental group were significantly lower than those in the blank group and the Lv-GFP group ( $<<0.05$; Fig. 5B, GATA4); the same trend was also demonstrated for the GATA4 bound with DNMT-3a ( $<<0.05$; Fig. 5C, GATA4). Almost no DNMT-3b binding was detected on the GATA4 and Nkx2.5 promoter region (Fig. 5D). In addition, DNMT-1 and DNMT-3a were demonstrated to bind to the $\mathrm{Nkx} 2.5$ promoter region, and the level of binding following Islet-1 infection was not significantly different compared with the blank group ( $\mathrm{P}>0.05$; Fig. 5B and $\mathrm{C}$, $\mathrm{Nkx} 2.5$, respectively). These results indicated that Islet-1 could reduce the DNMT-1 expression level and thus reduce its binding to the GATA4 promoter region. Eventually, the DNA methylation levels in the GATA4 promoter region decreased and GATA4 expression was promoted. However, DNMT-1 did not affect $\mathrm{Nkx} 2.5$ expression.

\section{Discussion}

The process of mesenchymal stem cell differentiation into cardiomyocytes is regulated by many factors, including intercellular interaction, signal pathway, epigenetics and paracrine (24-26). Studies have demonstrated that epigenetic modifications, such as histone acetylation and DNA methylation serve important roles in this process (27). Histone acetylation is the process by which the lysine residues within the N-terminal tail protruding from the histone core of the nucleosome are acetylated to determine the transcriptional 
$\mathbf{A}$
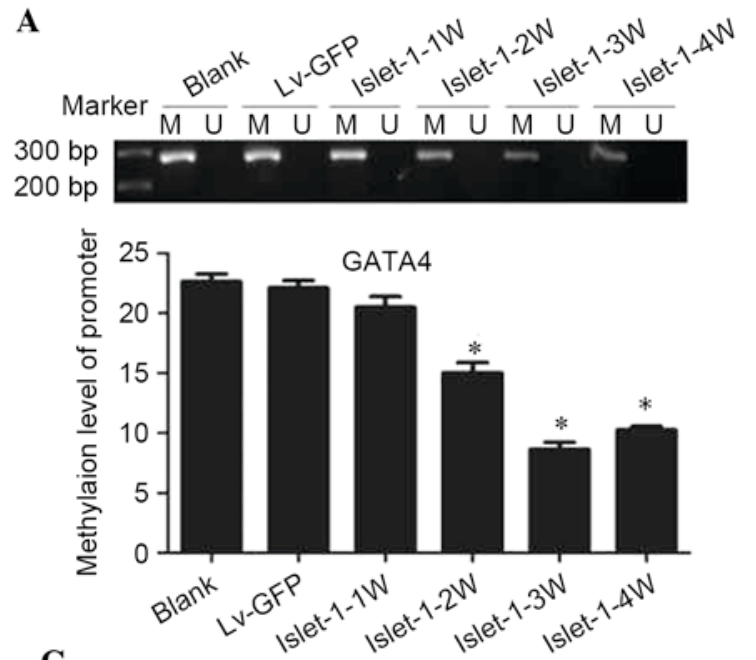

C

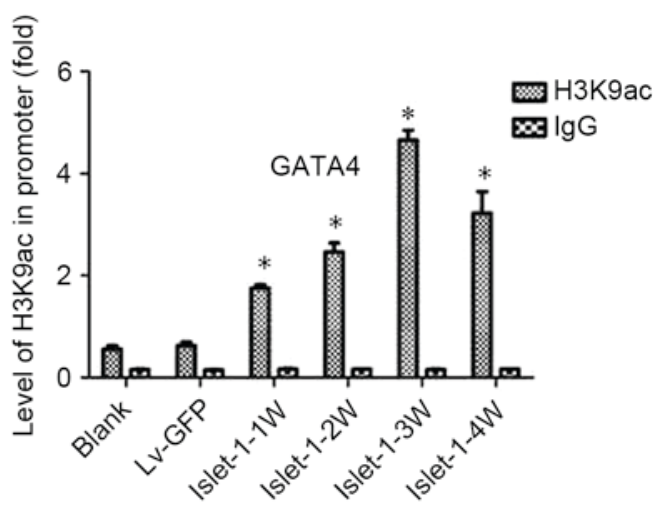

B
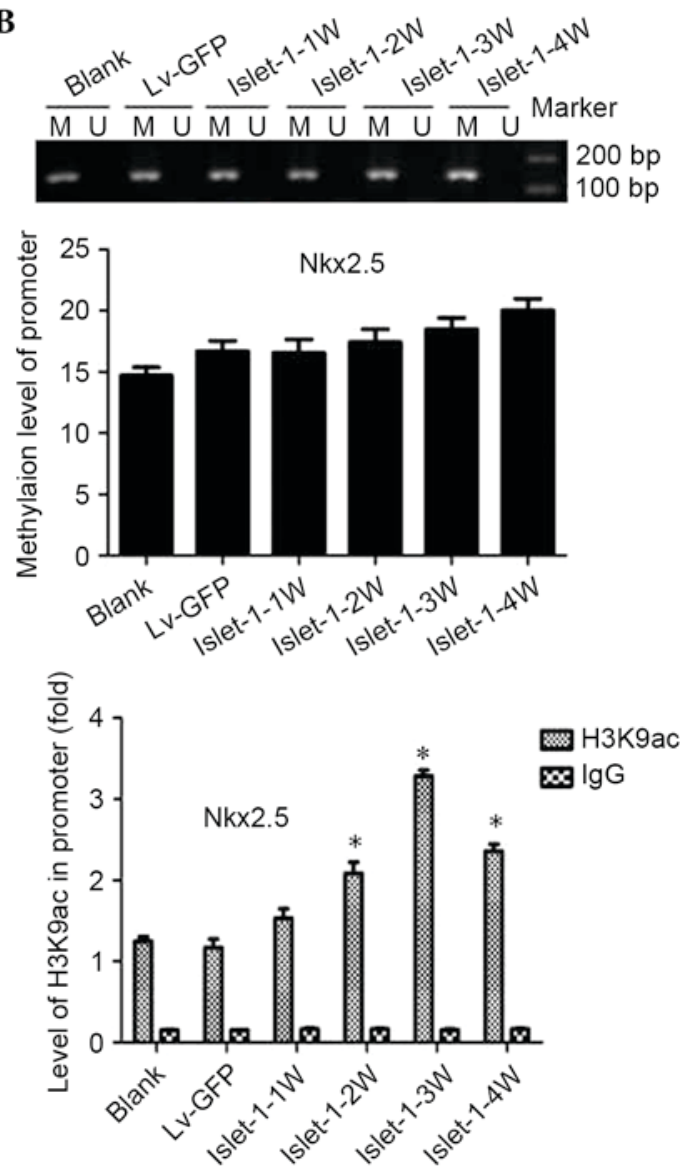

Figure 3. DNA methylation levels and acetylation levels of the histone H3K9 site in the GATA4 and Nkx2.5 promoter regions during the differentiation process promoted by Islet-1. (A) The detection of methylation levels on the GATA4 promoter (1329-1489 bp) by MSP assay. (B) The detection of the methylation levels at the Nkx2.5 promoter (51-219 bp) by MSP assay. (C) ChIP results demonstrated the levels of histone acetylation on the promoter regions of GATA4 and $\mathrm{Nkx} 2.5$. ${ }^{*} \mathrm{P}<0.05$ vs. blank group. GATA4, GATA binding protein 4; Nkx2.5, NK2 homeobox 5; MSP, methylation-specific polymerase chain reaction; Lv-GFP, lentiviral vector containing green fluorescent protein; Lv-islet-1, lentiviral vector containing Islet-1; M, methylated; U, unmethylated; 1 W, 1 week; 2 W, 2 weeks; 3 W, 3 weeks; 4 W, 4 weeks.

activity of chromatin (28), while DNA methylation is a process by which methylation modifications are added to alter the function of the DNA that is critical in the regulation of gene expression (29). A previous study from this group suggested the differentiation of stem cells into cardiomyocyte-like cells promoted by Islet-1 (13). The current study focused on two epigenetic modification methods: Histone acetylation and DNA methylation. The aim of the study was to elucidate which histone acetyltransferases and DNA methyltransferases could regulate the expression of specific early-stage transcription factors in cardiomyocytes and promote the differentiation of MSCs into cardiomyocyte-like cells.

The role of histone acetylation in early development and differentiation is a current topic of interest $(30,31)$. Regulation by this modification primarily occurs through HATs. The major function of HATs is to perform acetylation modification of the lysine residue at the amino terminus of the chromatin core histones, thereby loosening the chromatin structure and increasing the gene transcription activities (32). The first discovered histone acetyltransferase, Gcn5, primarily modifies nucleosomal histones and the free histones $\mathrm{H} 3$ and $\mathrm{H} 4$ (33-35). P300 is a coactivator and HAT that modifies 4 histones (H2A, H2B, H3 and H4) $(33,36,37)$. The present study demonstrated that during the Islet-1-induced differentiation of stem cells into cardiomyocyte-like cells, Gcn5 expression and its binding to the GATA4 and Nkx2.5 promoter regions both gradually increased. Conversely, the expression of P300 gradually decreased during the process of Islet-1-induced differentiation of stem cells into cardiomyocyte-like cells, and only a low level of binding was detected at the GATA4 and Nkx2.5 promoter regions. These results suggested that Islet-1 increased Gcn 5 expression to increase its binding to the Nkx2.5 and GATA4 promoter regions, enhance Nkx2.5 and GATA4 expression, and finally promote the differentiation of MSCs into cardiomyocyte-like cells.

DNA methylation is an important process in epigenetic modification, and is essential for normal development and stem cell differentiation. In mammalian cells, DNA methylation occurs mainly at the $\mathrm{C} 5$ position of $\mathrm{CpG}$ dinucleotides by DNA methyltransferase, which is a key enzyme in DNA methylation $(38,39)$. The main function of DNMT1 is to maintain the status and form of DNA methylation, whereas the main functions of DNMT3a and DNMT3b are to catalyze new DNA methylation sites and establish new methylation patterns (40). The results of the present study demonstrated a decrease in the methylation of $\mathrm{CpG}$ sites on the GATA4 promoter during the differentiation of $\mathrm{C} 3 \mathrm{H} 10 \mathrm{~T} 1 / 2$ cells into cardiomyocyte-like cells induced by Islet-1, while this process was negatively 
A
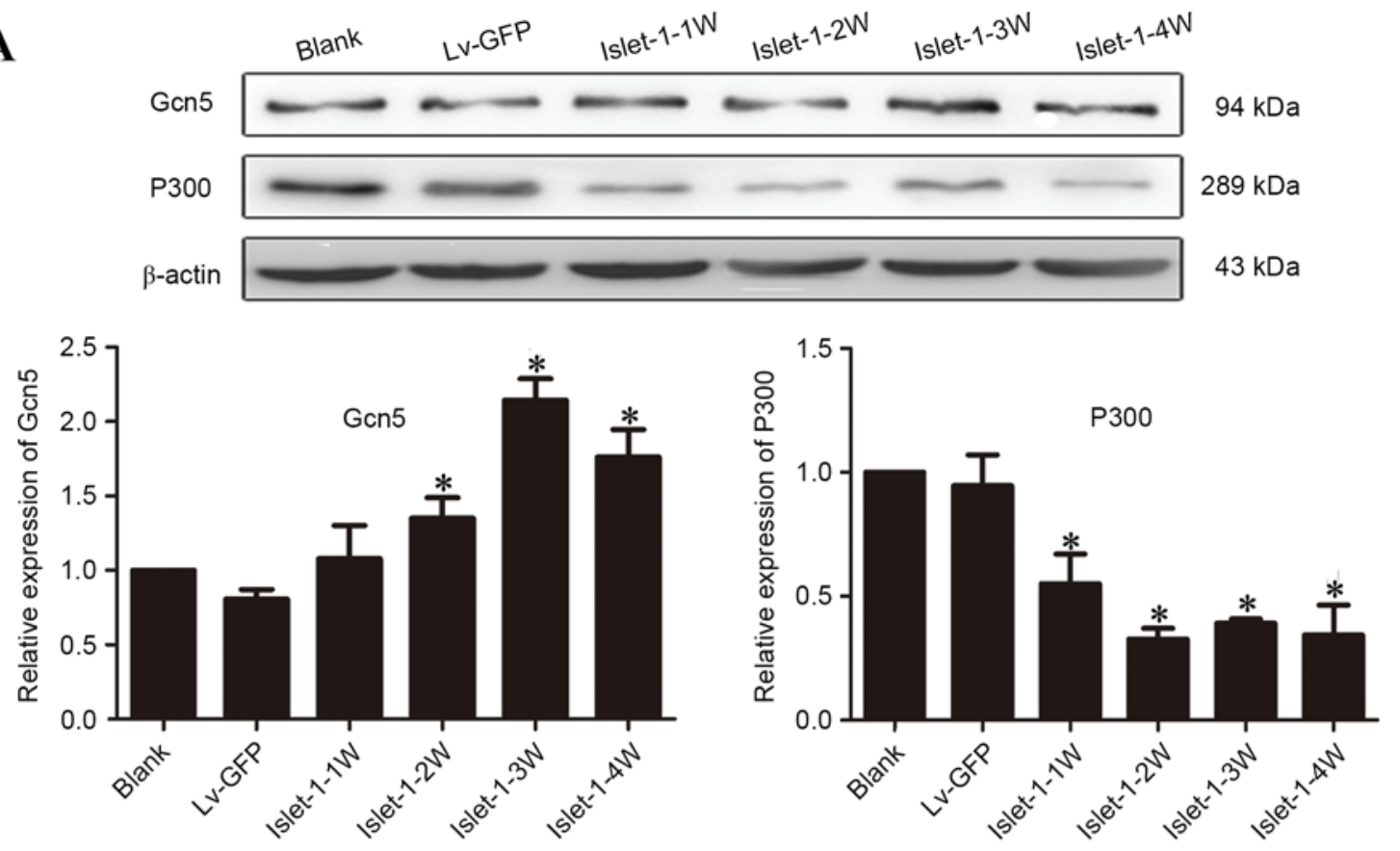

B
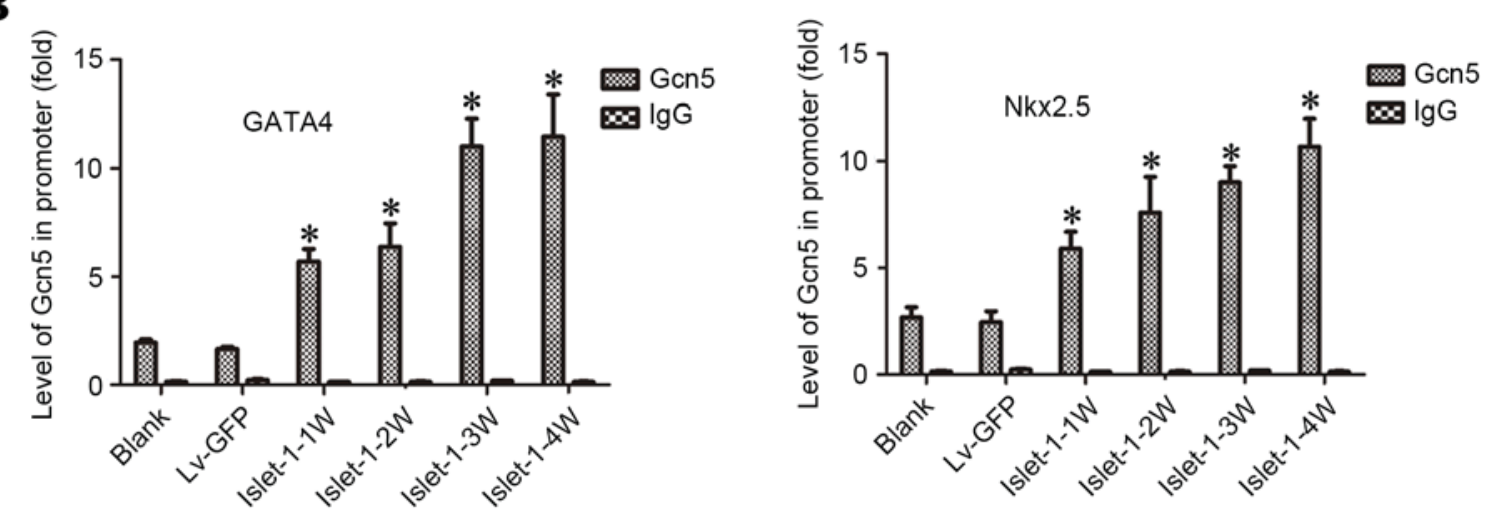

C
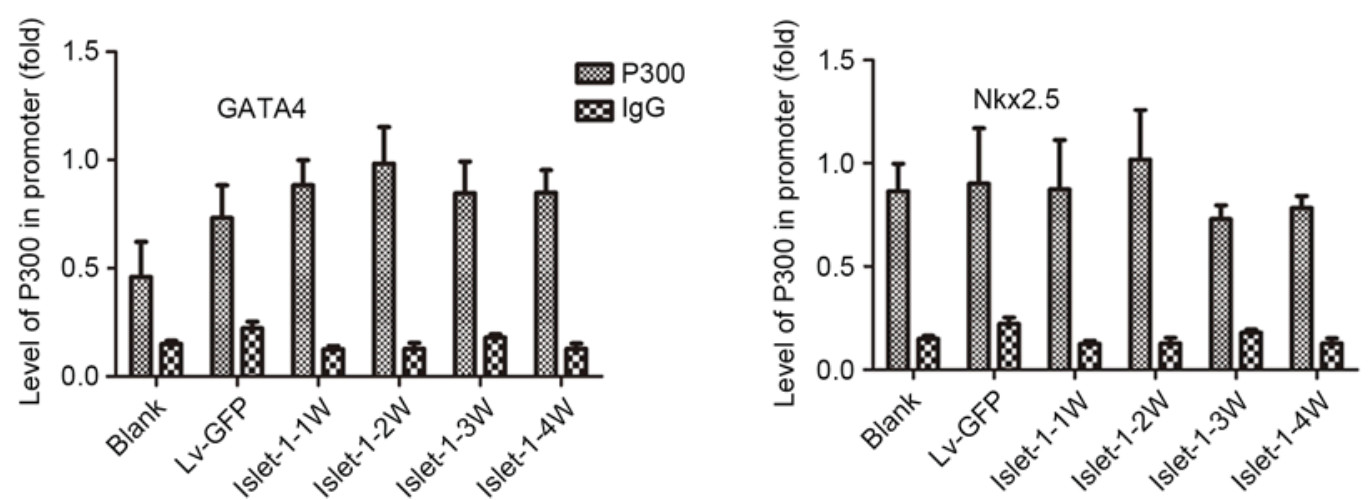

Figure 4. Detection of HATs on the histone H3K9 site that regulate the promoter regions of GATA4 and Nkx2.5. (A) Western blot analysis of Gcn5 and P300 HATs, with quantification relative to $\beta$-actin. (B) ChIP analysis of Gen5 bound to the GATA4 and Nkx2.5 promoter regions. (C) ChIP analysis of P300 bound to the GATA4 and Nkx2.5 promoter regions. "P<0.05 vs. blank control. HATS, histone acetyltransferases; GATA4, GATA binding protein 4; Nkx2.5, NK2 homeobox 5; Lv-GFP, lentiviral vector containing green fluorescent protein; Lv-islet-1, lentiviral vector containing Islet-1; $1 \mathrm{~W}, 1$ week; 2 W, 2 weeks; 3 W, 3 weeks; 4 W, 4 weeks; Gcn5, general control of amino acid biosynthesis protein 5; ChIP, chromatin immunoprecipitation.

associated with the GATA4 mRNA expression level. In addition, DNMT-1 expression and its binding to GATA4 both gradually decreased during the Islet- 1 induced differentiation of stem cells into cardiomyocyte-like cells. Although DNMT-3a expression gradually increased, the binding level to the GATA4 promoter was decreased. DNMT-3b expression and its binding to GATA4 and Nkx2.5 were almost undetectable. Furthermore, it was observed that, although DNMT-1 bound to $\mathrm{Nkx} 2.5$, the level of binding did not become altered during the differentiation process. The authors speculated that 
A
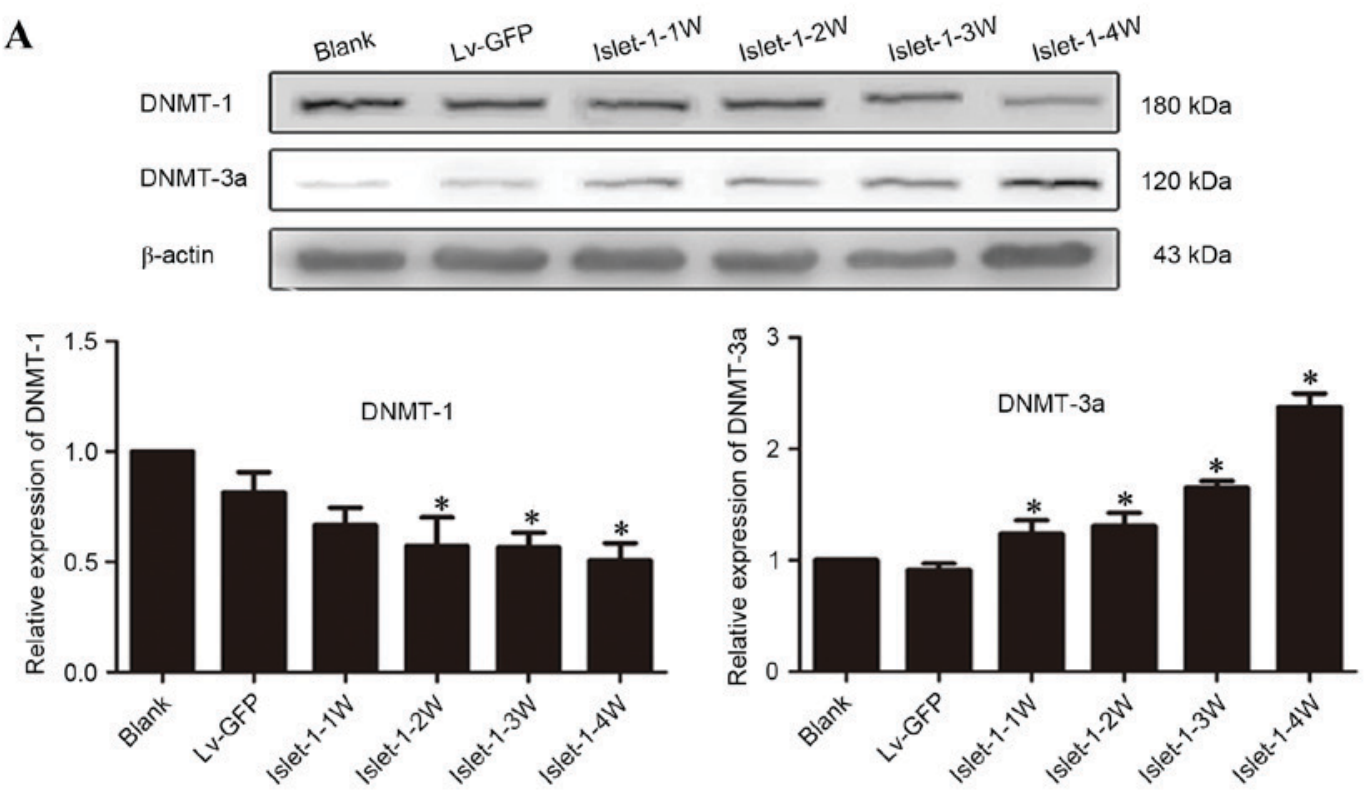

B
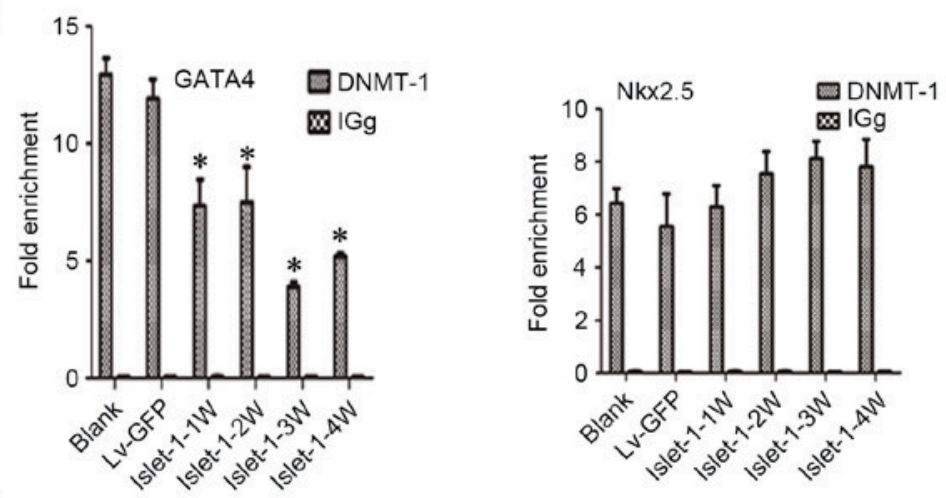

C
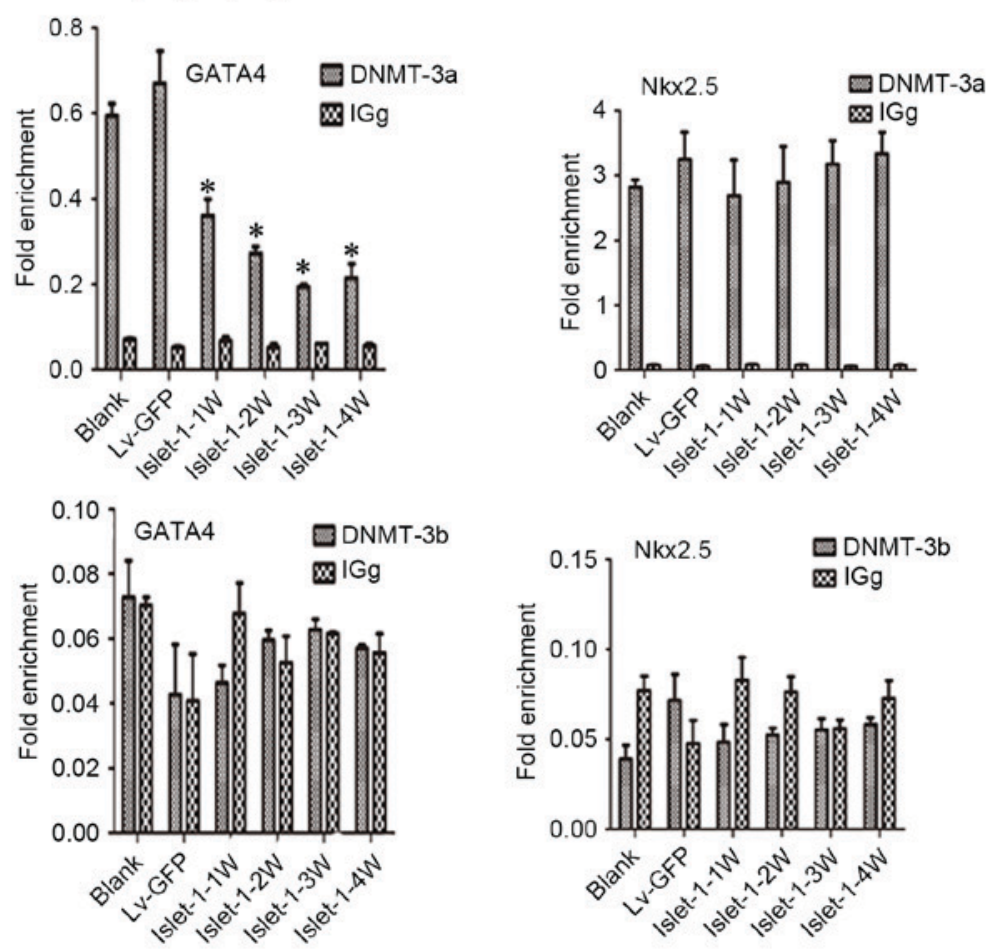

Figure 5. Detection of DNMTs that regulate the GATA4 promoter region. (A) Western blot analysis of DNMT-1 and DNMT-3a expression, with quantification relative to $\beta$-actin. (B) ChIP analysis of DNMT-1 bound to the GATA4 and Nkx2.5 promoter regions. (C) ChIP analysis of DNMT-3a bound to the GATA4 and Nkx2.5 promoter regions. (D) ChIP analysis of DNMT-3b bound to the GATA4 and Nkx2.5 promoter regions. "P<0.05 vs. blank control. DNMT, DNA methyltransferase; GATA4, GATA binding protein 4; Nkx2.5, NK2 homeobox 5; Lv-GFP, lentiviral vector containing green fluorescent protein; Lv-islet-1, lentiviral vector containing Islet-1; 1 W, 1 week; 2 W, 2 weeks; 3 W, 3 weeks; 4 W, 4 weeks; ChIP, chromatin immunoprecipitation. 
Islet-1 decreased DNMT-1 expression to reduce its binding to GATA4 and caused the gradual reduction of the methylation level of the GATA4 gene, thereby increasing GATA4 gene expression. There was no association between the binding level of DNMT-1 in Nkx2.5 promoter and the expression of $\mathrm{Nkx} 2.5$, which suggested that $\mathrm{Nkx} 2.5$ was not regulated by DNA methylation in the process.

A previous study has identified links between DNA methylation and histone hypoacetylation (41). In the present study, the histone acetylation level on the GATA4 promoter presented a gradual increasing trend that was positively correlated with the mRNA level. In addition, the histone acetylation level on the $\mathrm{Nkx} 2.5$ promoter was consistent with its expression level and showed a gradual increasing trend. However, the methylation level of $\mathrm{CpG}$ sites on the $\mathrm{Nkx} 2.5$ promoter did not significantly alter during the differentiation process. Therefore, it was concluded that DNA methylation and histone acetylation concurrently participated in the regulation of GATA4 expression during the Islet-1-induced differentiation of $\mathrm{C} 3 \mathrm{H} 10 \mathrm{~T} 1 / 2$ cells into cardiomyocyte-like cells. In contrast, $\mathrm{Nkx} 2.5$ expression may not be affected by DNA methylation. These results indicated that DNA methylation did not regulate the expression of all genes and thus exhibited selectivity. Furthermore, histone acetylation levels and DNA methylation levels had opposing trends with GATA4 expression. Previous studies have reported that epigenetic modifications influenced one another during the regulation of gene expression (42). Therefore, these two modifications may have interactive functions during the regulation of GATA4 expression. However, this hypothesis requires further study for validation.

In summary, the present study confirmed that histone acetylation and DNA methylation participated in the regulation of the early specific gene GATA4 in cardiomyocytes through Gen5 and DNMT-1 during the Islet-1-induced differentiation of MSCs into cardiomyocytes. However, the Nkx2.5 expression appeared to be regulated by Gen5 instead of DNA methylation. Furthermore, it was observed that these two epigenetic modifications had a specific relationship. Future studies are required to clarify whether there is association between them and to elucidate the mechanism underlying their interaction. The current study preliminarily proposed the mechanism underlying the promotion of MSCs differentiation into cardiomyocyte-like cells based on the histone acetylation and DNA methylation induced by Islet-1. These results provided an important experimental basis for future studies on the function of epigenetic modifications in MSCs differentiation and novel insights into the study of the specific differentiation of MSCs.

\section{Acknowledgements}

This study was supported by the National Natural Science Foundation of China (grant no. 81370261).

\section{References}

1. Chou SH, Lin SZ, Kuo WW, Pai P, Lin JY, Lai CH, Kuo CH, Lin KH, Tsai FJ and Huang CY: Mesenchymal stem cell insights: Prospects in cardiovascular therapy. Cell Transplant 23: 513-529, 2014.
2. Kuraitis D, Ruel M and Suuronen EJ: Mesenchymal stem cells for cardiovascular regeneration. Cardiovasc Drugs Ther 25: 349-362, 2011.

3. Bianco P, Robey PG and Simmons PJ: Mesenchymal stem cells: Revisiting history, concepts and assays. Cell Stem Cell 2: 313-319, 2008.

4. Aldahmash A, Zaher W, Al-Nbaheen M and Kassem M: Human stromal (mesenchymal) stem cells: Basic biology and current clinical use for tissue regeneration. Ann Saudi Med 32: 68-77, 2012.

5. Huang L, Ma W, Ma Y, Feng D, Chen H and Cai B: Exosomes in mesenchymal stem cells, a new therapeutic strategy for cardiovascular diseases? Int J Biol Sci 11: 238-245, 2015.

6. Sheng CC, Zhou L and Hao J: Current stem cell delivery methods for myocardial repair. Biomed Res Int 2013: 547902, 2013.

7. Brade T, Gessert S, Kühl M and Pandur P: The amphibian second heart field: Xenopus islet-1 is required for cardiovascular development. Dev Biol 311: 297-310, 2007.

8. Bu L, Jiang X, Martin-Puig S, Caron L, Zhu S, Shao Y, Roberts DJ, Huang PL, Domian IJ and Chien KR: Human ISL1 heart progenitors generate diverse multipotent cardiovascular cell lineages. Nature 460: 113-117, 2009.

9. Yang L, Cai CL, Lin L, Qyang Y, Chung C, Monteiro RM, Mummery CL, Fishman GI, Cogen A and Evans S: Isl1Cre reveals a common Bmp pathway in heart and limb development. Development 133: 1575-1585, 2006.

10. Laugwitz KL, Moretti A, Caron L, Nakano A and Chien KR: Islet1 cardiovascular progenitors: A single source for heart lineages? Development 135: 193-205, 2008.

11. Nakano A, Nakano H and Chien KR: Multipotent islet-1 cardiovascular progenitors in development and disease. Cold Spring Harb Symp Quant Biol 73: 297-306, 2008.

12. Cai CL, Liang X, Shi Y, Chu PH, Pfaff SL, Chen J and Evans S: Isl1 identifies a cardiac progenitor population that proliferates prior to differentiation and contributes a majority of cells to the heart. Dev Cell 5: 877-889, 2003.

13. Yin N, Lu R, Lin J, Zhi S, Tian J and Zhu J: Islet-1 promotes the cardiac-specific differentiation of mesenchymal stem cells through the regulation of histone acetylation. Int J Mol Med 33: 1075-1082, 2014.

14. Li L, Zhu J, Tian J, Liu X and Feng C: A role for Gen5 in cardiomyocyte differentiation of rat mesenchymal stem cells. Mol Cell Biochem 345: 309-316, 2010.

15. Peng C, Zhu J, Sun HC, Huang XP, Zhao WA, Zheng M, Liu LJ and Tian J: Inhibition of histone $\mathrm{H} 3 \mathrm{~K} 9$ acetylation by anacardic acid can correct the over-expression of Gata4 in the hearts of fetal mice exposed to alcohol during pregnancy. PLoS One 9: e104135, 2014.

16. Bird A: DNA methylation patterns and epigenetic memory. Genes Dev 16: 6-21, 2002.

17. Ting AH, Jair KW, Suzuki H, Yen RW, Baylin SB and Schuebel KE: Mammalian DNA methyltransferase 1: Inspiration for new directions. Cell Cycle 3: 1024-1026, 2004.

18. Wu Y, Strawn E, Basir Z, Halverson G and Guo SW: Aberrant expression of deoxyribonucleic acid methyltransferases DNMT1, DNMT3A and DNMT3B in women with endometriosis. Fertil Steril 87: 24-32, 2007.

19. Luczak MW, Roszak A, Pawlik P, Kędzia H, Kędzia W, Malkowska-Walczak B, Lianeri M and Jagodziński PP: Transcriptional analysis of CXCR4, DNMT3A, DNMT3B and DNMT1 gene expression in primary advanced uterine cervical carcinoma. Int J Oncol 40: 860-866, 2012.

20. Liao J, Karnik R, Gu H, Ziller MJ, Clement K, Tsankov AM, Akopian V, Gifford CA, Donaghey J, Galonska C, et al: Targeted disruption of DNMT1, DNMT3A and DNMT3B in human embryonic stem cells. Nat Genet 47: 469-478, 2015.

21. Livak KJ and Schmittgen TD: Analysis of reltive gene expression data using real-time quantitative PCR and the 2(-Delta Delta C(T)) method. Methods 25: 402-408, 2001.

22. Fu L, Xia Y, He J, Liu X, Chen X, Wang Y and Ding Y: Data analysis and its analytical softs application on DNA methylation in tumor research. Zhong Qing Yi Xue Bian Ji Bu 41: 1719-1721, 1726, 2012 (In Chinese).

23. Xu H, Yi Q, Yang C, Wang Y, Tian J and Zhu J: Histone modifications interact with DNA methylation at the GATA4 promoter during differentiation of mesenchymal stem cells into cardiomyocyte-like cells. Cell Prolif 49: 315-329, 2016.

24. Kawamura T, Ono K, Morimoto T, Wada H, Hirai M, Hidaka K, Morisaki T, Heike T, Nakahata T, Kita T and Hasegawa K: Acetylation of GATA-4 is involved in the differentiation of embryonic stem cells into cardiac myocytes. J Biol Chem 280: 19682-19688, 2005. 
25. Pasini A, Bonafè F, Govoni M, Guarnieri C, Morselli PG, Sharma HS, Caldarera CM, Muscari C and Giordano E: Epigenetic signature of early cardiac regulatory genes in native human adipose-derived stem cells. Cell Biochem Biophys 67: $255-262,2013$

26. Zhang $\mathrm{H}$ and Wang ZZ: Mechanisms that mediate stem cell self-renewal and differentiation. J Cell Biochem 103 709-718, 2008.

27. Ohtani K and Dimmeler S: Epigenetic regulation of cardiovascular differentiation. Cardiovasc Res 90: 404-412, 2011.

28. Spivakov M and Fisher AG: Epigenetic signatures of stem-cell identity. Nat Rev Genet 8: 263-271, 2007.

29. Hawkins RD, Hon GC, Lee LK, Ngo Q, Lister R, Pelizzola M, Edsall LE, Kuan S, Luu Y, Klugman S, et al: Distinct epigenomic landscapes of pluripotent and lineage-committed human cells. Cell Stem Cell 6: 479-491, 2010.

30. Horikoshi M: Histone acetylation: From code to web and router via intrinsically disordered regions. Curr Pharm Des 19: 5019-5042, 2013.

31. Oligny LL: Human molecular embryogenesis: An overview. Pediatr Dev Pathol 4: 324-343, 2001.

32. Sadoul K, Boyault C, Pabion M and Khochbin S: Regulation of protein turnover by acetyltransferases and deacetylases. Biochimie 90: 306-312, 2008.

33. Kurdistani SK and Grunstein M: Histone acetylation and deacetylation in yeast. Nat Rev Mol Cell Biol 4: 276-284, 2003.

34. Hu Z, Song N, Zheng M, Liu X, Liu Z, Xing J, Ma J, Guo W, Yao Y, Peng H, et al: Histone acetyltransferase GCN5 is essential for heat stress-responsive gene activation and thermotolerance in arabidopsis. Plant J 84: 1178-1191, 2015.

35. Kuo YM, Andrews AJ: Quantitating the specificity and selectivity of Gen5-mediated acetylation of histone H3. PLoS One 8: e54896, 2013.
36. Kornacki JR, Stuparu AD and Mrksich M: Acetyltransferase p300/CBP associated factor (PCAF) regulates crosstalk-dependent acetylation of histone $\mathrm{H} 3$ by distal site recognition. ACS Chem Biol 10: 157-164, 2015

37. Zheng M, Zhu J, Lu T, Liu L, Sun H, Liu Z and Tian J: P300-mediated histone acetylation is essential for the regulation of GATA4 and MEF2C by BMP2 in $\mathrm{H} 9 \mathrm{c} 2$ cells. Cardiovase Toxicol 13: 316-322, 2013

38. Nagre NN, Subbanna S, Shivakumar M, Psychoyos D and Basavarajappa BS: CB1-receptor knockout neonatal mice are protected against ethanol-induced impairments of DNMT1, DNMT3A and DNA methylation. J Neurochem 132: 429-442, 2015.

39. Arakawa Y, Watanabe M, Inoue N, Sarumaru M, Hidaka Y and Iwatani Y: Association of polymorphisms in DNMT1, DNMT3A, DNMT3B, MTHFR and MTRR genes with global DNA methylation levels and prognosis of autoimmune thyroid disease. Clin Exp Immunol 170: 194-201, 2012.

40. Subramaniam D, Thombre R, Dhar A and Anant S: DNA methyltransferases: A novel target for prevention and therapy. Front Oncol 4: 80, 2014.

41. Minardi D, Lucarini G, Filosa A, Zizzi A, Milanese G, Polito M Jr, Polito M, Di Primio R, Montironi R and Muzzonigro G: Do DNA-methylation and histone acetylation play a role in clear cell renal carcinoma? Analysis of radical nephrectomy specimens in a long-term follow-up. Int J Immunopathol Pharmacol 24: 149-158, 2011.

42. Wu LP, Wang X, Li L, Zhao Y, Lu S, Yu Y, Zhou W, Liu X, Yang J, Zheng Z, et al: Histone deacetylase inhibitor depsipeptide activates silenced genes through decreasing both $\mathrm{CpG}$ and H3K9 methylation on the promoter. Mol Cell Biol 28: 3219-3235, 2008. 